\title{
Design and simulation of multilayer hybrid foam material for acoustic application
}

\author{
L. Yuvaraj ${ }^{1, *}$, S. Jeyanthi ${ }^{2}$, Lenin Babu Mailan Chinnapandi ${ }^{3}$, and Elammaran Jayamani ${ }^{4}$ \\ ${ }^{1}$ Department of Automobile Engineering, Acharya Institute of Technology, Bangalore, India \\ 2 School of Mechanical and Building Sciences, Vellore Institute of Technology, Chennai 600127, India \\ ${ }^{3}$ Electric Vehicles Incubation, Testing and Research Centre, Vellore Institute of Technology Chennai, India \\ 4 Swinburne University of Technology Sarawak Campus, Malaysia
}

Received: 10 January 2021 / Accepted: 7 July 2021

\begin{abstract}
New acoustic multilayer absorber fabricated by coupling closed-cell metallic foam and open-cell polymeric foam, which aimed to develop a practical use of metallic foam in the noise control application. In prior, the individual sound absorption coefficient of both foam materials with different thicknesses measured by the impedance tube method as per ASTM E-1050. Using inverse characterization technique, the intrinsic properties needed for five parameter models in a numerical study are predicted. The measured characteristic impedance, complex wave propagation, and sound absorption coefficient of the individual foams are in close agreement with the prediction. Subsequently, a different configuration of multilayer absorber is modeled using obtained properties, and their acoustic performance is evaluated. The result indicates that the coupling of polymeric foam with metallic one exhibits enhanced sound absorption and usage of closed-cell metallic foam in noise control material. Furthermore, the result demonstrates that absorption capability entirely relies on the placement of polymeric foam in the configuration. The proposed hybrid multilayer absorber coupled with test bench car for interior acoustic study, where $5-30 \mathrm{~dB}$ is reduction is noticed in $1 / 3$ rd octave plot.
\end{abstract}

Keywords: Aluminium foam / polymeric foam / sound absorption / particle swarm optimization / test bench car

\section{Introduction}

Lightweight foamed materials are produced from ceramics, metals, and polymers, used in many engineering applications due to high energy absorption and strength to weight ratio. Based on the connectivity between the cells, the foam can be classified into an open-cell and a closed-cell. Mostly the aluminum foam that is available in commercial is closed cell foam. The sound absorption characteristic of closedcell foam is good only at higher frequency regions [1]. The main reason is that the metallic frame is stiffer than the surrounding fluid, so the acoustic losses viscous and thermal occur at metal-fluid interface limited by isolated closed cells. Also, it depends on the closed pore size, shape, and structure. Extensive research was carried out in polymeric foam, which is fully reticulated, partially reticulated, and completely closed to obtain the acoustic properties [2-4]. These materials have a wide range of acoustic properties depending on poroelastic nature, pore

\footnotetext{
* e-mail: mechanicalyuva5236@gmail.com
}

size, and reticulation rate. Sound absorption of open-cell and partial reticulated foam exhibits good viscous and thermal losses effect.To overcome the drawback of aluminium foam, Sound absorption of high-density material can be enhanced by the combination of lowdensity material as a multilayer porous material [5] which acts as a mass-spring system leads to additional attenuation of sound.

Two critically linked membrane-type acoustic metamaterials sandwiching a porous material layer were proposed by Wang et al. [6] as a layered porous material. Adaptive algorithms are used to detect the radiating source which is not familiar with weighted classical array of sensors [7]. The study reported that the material characterization is used to design resonator by optimization process [8]. Also, that the non-acoustic parameter needed for numerical simulation can be predicted using inverse characterisation with different algorithm for instance particle swarm optimization algorithm (PSO) [9], genetic algorithm [10] and so on. The sound absorption coefficient, especially the low frequency sound absorption coefficient is increased obviously as the growing of samples 
Table 1. Properties of Al foam.

\begin{tabular}{llllll}
\hline Thickness & Pore size & Porosity & Cell type & Density & Compressive strength \\
\hline $10 \mathrm{~mm}$ & $2-11 \mathrm{~mm}$ & $60-90 \%$ & Closed cell & $0.75 \mathrm{~g} / \mathrm{cm}^{3}$ & $3-17 \mathrm{Mpa}$ \\
\hline
\end{tabular}

Table 2. Foam preparation specification.

\begin{tabular}{ll}
\hline Characteristics & Specification \\
\hline Empeyol WSF 300/1-8(HLD) & $100 \mathrm{Pbw}$ \\
Wannate 8018 & $65 \mathrm{Pbw}$ \\
Cream time & $9 \mathrm{~s}$ \\
Gel time & $65 \mathrm{~s}$ \\
Rise time & $77 \mathrm{~s}$ \\
Demould time & $210 \mathrm{~s}$ \\
\hline
\end{tabular}

thickness. This phenomenon can be found in porous metal as well as flexible materials. The detailed finite element modeling presented in various studies by author and referred [10-12]. The present work demonstrates closed-cell metallic foam usage in the acoustic application by proposing new hybrid foam comprises closed cell metallic foam and open-cell polymeric foam as a multilayer porous absorber. The proposed configuration experimented in impedance tube and same is validated by numerical results. Furthermore, the best configuration is coupled with test bench car to evaluate sound pressure level using COMSOL multiphysics acoustic module.

\section{Materials and methods}

\subsection{Aluminum foam}

The metallic aluminium $(\mathrm{Al})$ foam procured from local source whose dimension ranges $0.1 \times 0.1 \times 0.01 \mathrm{~mm}^{3}$ and cutted into impedance diameter $96 \mathrm{~mm}$. The properties of Al foam depicted in Table 1.

\subsection{Polyurethane foam}

Flexible polyurethane is synthesized by isocyanate and polyol, and those chemicals duly supplied Manali Petrochemicals Limited (Chennai, India). EMPEYOL WSF $300 / 1-8$ (HLD) used as a polyol, which is a pre-mixture of all additives like a catalyst, stabilizer, water, chain extender, surfactants, and WANNATE 8018 as isocyanate. The properties of foam chemicals given in Table 2 .

\subsection{Hybrid foam}

Once the sample cut into the desired shape, placed in a circular die, and the polyol isocyanate mixture is poured. After the demould time, the sample is taken out to remove the additional thickness material also the rough surface of aluminum foam, making a strong bond when the molten

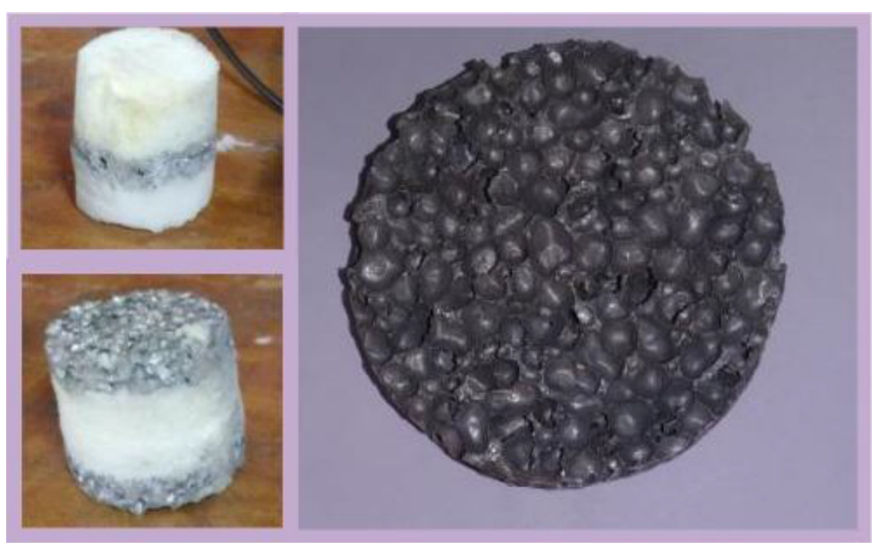

Fig. 1. Prepared hybrid foam.

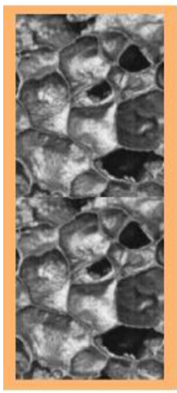

AL40

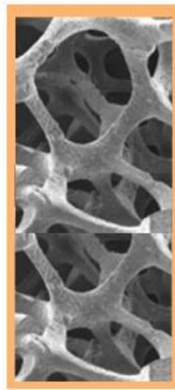

PU40

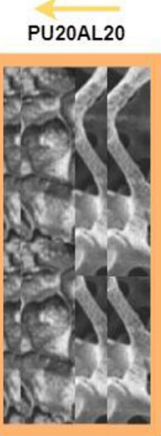

AL20PU20

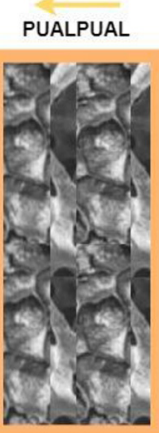

ALPUALPU
Fig. 2. Schematic diagram of a multilayer configuration.

polyol mixture cures. The prepared sample and its configuration is shown in Figures 1 and 2. The overall thickness of all configurations is $40 \mathrm{~mm}$ is maintained, and the sample name is given in the following ways as depicted in Table 3.

\subsection{Experimental testing}

The sound absorption coefficient of the individual sample and hybrid foam is tested in impedance as per ASTM E 1050 [13], where the frequency range of interest is $220 \mathrm{~Hz}$ to $4000 \mathrm{~Hz}$. Samples are cut into circular sections with a diameter of 33 and $100 \mathrm{~mm}$. Three samples for each category are used for this test. The white noise signal excited from source-channel DAQ connected to the power amplifier then to $16 \Omega$ speakers at one end and two $1 / 2^{\prime \prime}$ MicrotechGefell microphones connected to $\mathrm{M}+\mathrm{P}$ Vibpilot 
Table 3. Sample nomenclature.

\begin{tabular}{ll}
\hline Sample & Overall thickness $(40 \mathrm{~mm})$ \\
\hline AL40 & Aluminum foam $40 \mathrm{~mm}$ \\
PU40 & Polyurethane foam $40 \mathrm{~mm}$ \\
AL20PU20 & Al foam $20 \mathrm{~mm}+\mathrm{Pu}$ foam $20 \mathrm{~mm}$ \\
PU20AL20 & $\mathrm{Pu}$ foam $20 \mathrm{~mm}+\mathrm{Al}$ foam $20 \mathrm{~mm}$ \\
ALPUALPU & $\mathrm{Al} 10 \mathrm{~mm}+\mathrm{Pu}$ foam $10 \mathrm{~mm}+\mathrm{Al}$ foam $10 \mathrm{~mm}+\mathrm{Pu}$ foam $10 \mathrm{~mm}$ \\
PUALPUAL & $\mathrm{Pu}$ foam $10 \mathrm{~mm}+\mathrm{Al}$ foam $10 \mathrm{~mm}+\mathrm{Pu}$ foam $10 \mathrm{~mm}+\mathrm{Al}$ foam $10 \mathrm{~mm}$ \\
\hline
\end{tabular}

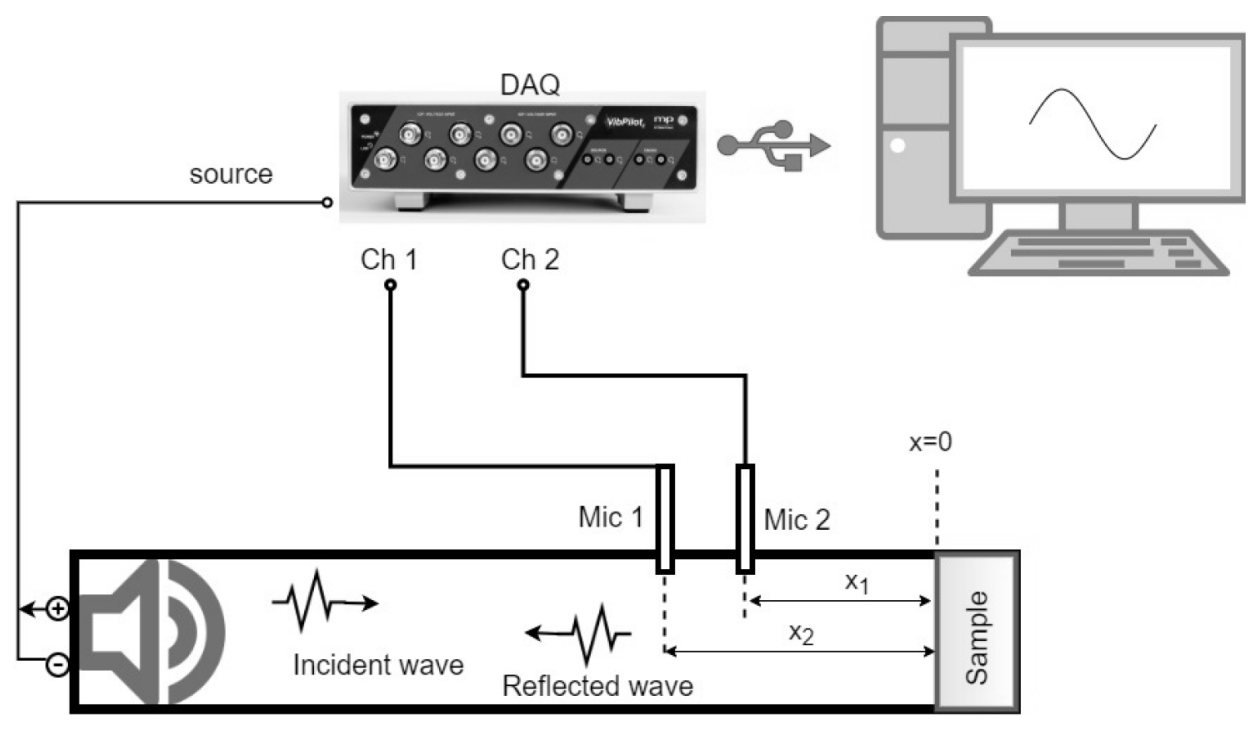

Fig. 3. Schematic diagram of impedance tube.

DAQ. Using the $\mathrm{M}+\mathrm{P}$ spectrum analyzer, the time domain was extracted of useful bandwidth $8196 \mathrm{~Hz}$ with a frequency resolution of $0.5 \mathrm{~Hz}$, which is imported to MATLAB ${ }^{\circledR}$ to calculate transfer function between two microphones. The schematic diagram of the impedance tube is shown in Figure 3.

$$
\left(K_{0}\right)=\frac{2 \pi f}{C_{0}}
$$

where $K_{0}$ the wavenumber, $f$ is the frequency, and $C_{0}$ is the speed of sound

$$
\left(H_{12}\right)=\frac{p_{2}}{p_{1}}=\frac{e^{j k_{0} x_{2}}+r e^{-j k_{0} x_{2}}}{e^{j k_{0} x_{1}}+r e^{-j k_{0} x_{1}}}
$$

where $H_{12}$ is the acoustic transfer function, $\mathrm{p}_{1}$ and $\mathrm{p}_{2}$ are the measured acoustic pressure of the two microphones, and $x_{1}$ and $x_{2}$ are the distance between the reference plane (sample position of $x=0$ ) and the two microphones. Finally, the reflection coefficient and sound absorption co-efficient $\alpha$ is obtained by

$$
\begin{gathered}
R=\frac{e^{-j k S}-H_{12}}{H_{12}-e^{j k S}} \times e^{2 j k(l+S)} \\
(\alpha)=1-|R|^{2}
\end{gathered}
$$

\subsection{Inverse characterization using PSO}

The PSO works on the social sharing of information of organism among the fish in a flock. While moving into the community, fish exchange information about theirposition, velocity, and fitness function value. In the PSO algorithm, the words fish andparticle are used interchangeably. Individual particle provides information about itsposition vector in the solution space and the associated velocity vector. All particleschange their position at a particular time set in the algorithm. Then the velocity vector data isused to calculate the new location for the next time step. The PSO algorithmrecords the information about the best individual position attained by each particle known as $p^{\text {best }}$ and the best global position achieved by all particles together knownas $G^{\text {best }}$. In each iteration, $G^{\text {best }}$ represents a least error with the measured value and all $p^{\text {best }}$ values follow $G^{\text {best }}$ values to give the best solution. Hence, with the knowledge ofthese two best values, all particles collectively move towards the optimum globalposition.In this optimization problem, the design variables are the non-acousticalparameters. The non-acoustical parameters for the Johnson-Champoux-Allard (JCA) equivalent fluid modelwith a rigid frame model are porosity, Tortuosity, flow resistivity, viscous characteristic length (VCL), and thermal characteristic length (TCL). The objective 
Table 4. Constraint for the design variable.

\begin{tabular}{llllll}
\hline Material properties & Porosity & Flow Resistivity $\left(\mathrm{Ns} / \mathrm{m}^{4}\right)$ & Tortuosity & VCL $(\mu \mathrm{m})$ & TCL $(\mu \mathrm{m})$ \\
\hline Lower bounds & 0 & 1000 & 1 & 0 & 0 \\
Upper bounds & 1 & 200000 & 3 & 1000 & 1000 \\
\hline
\end{tabular}
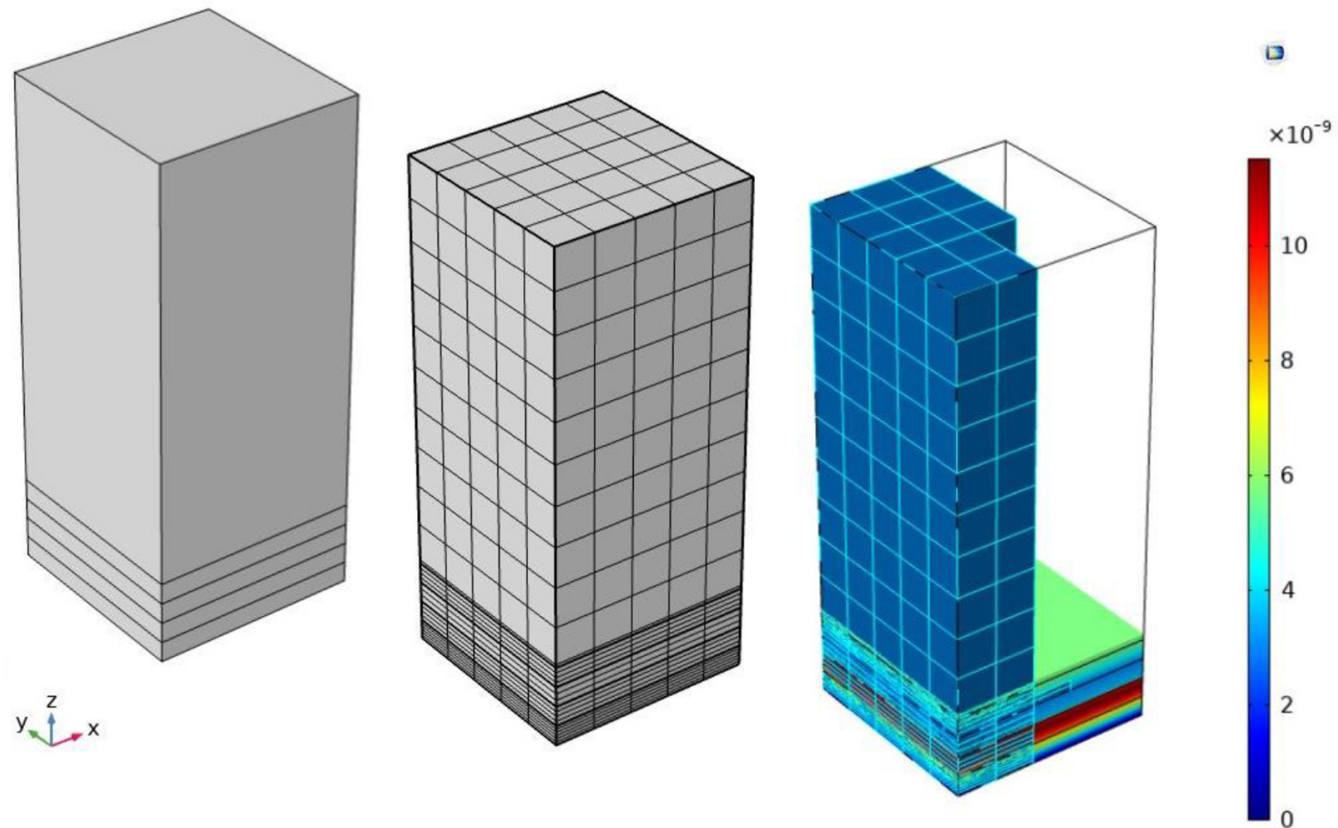

Fig. 4. Numerical simulation of four-layer hybrid foam.

Table 5. Predicted non-acoustic parameters using PSO algorithm.

\begin{tabular}{llllll}
\hline Sample & \multicolumn{5}{c}{ Non-acoustic parameters } \\
\cline { 2 - 6 } & Porosity & Flow Resistivity $\left(\mathrm{Ns} / \mathrm{m}^{4}\right)$ & Tortuosity & VCL $(\mu \mathrm{m})$ & TCL $(\mu \mathrm{m})$ \\
\hline AL40 & 0.87 & 38816 & 2.46 & 548 & 548 \\
PU40 & 0.96 & 11965 & 1.79 & 66 & 286 \\
AL20PU20 & 0.85 & 53944 & 1.48 & 26 & 89 \\
PU20AL20 & 0.98 & 45387 & 1.08 & 13 & 274 \\
ALPUALPU & 0.86 & 29681 & 1.55 & 69 & 144 \\
PUALPUAL & 0.97 & 15373 & 1.93 & 78 & 286 \\
\hline
\end{tabular}

function used here is defined by equation (5)

$$
E(\alpha)=\left[\frac{\sqrt{\frac{1}{n} \sum_{i=1}^{n}\left(d_{i}^{\exp }-d_{i}^{\mathrm{jca}}\right)^{2}}}{d_{\max }^{\exp }-d_{\min }^{\exp }}\right]
$$

where $E(\alpha)$ is the normalised means square error, $n$ is the total number of data, $d_{i}^{\exp }$ is the experimental sound absorption, $d_{i}^{\text {jca }}$ is the predicted sound absorption using JCA model. Constraints used for this study presented in Table 4.

\subsection{Numerical simulation}

\subsubsection{Multilayer simulation}

Sound absorption of multilayer configuration of hybrid foam analyzed numerically using COMSOL Multiphysics [7-9]. In component section, using the geometry option, a block created and the plane partitioned into 5 domains with the help of Boolean operation. The lengthier domain chosen as pressure acoustics module and air material property is applied. Similarly, four equal thicknesses are chosen poroelastic waves module and material property used as per configuration order. Multiphysics coupling is applied to make the interaction between porous and 


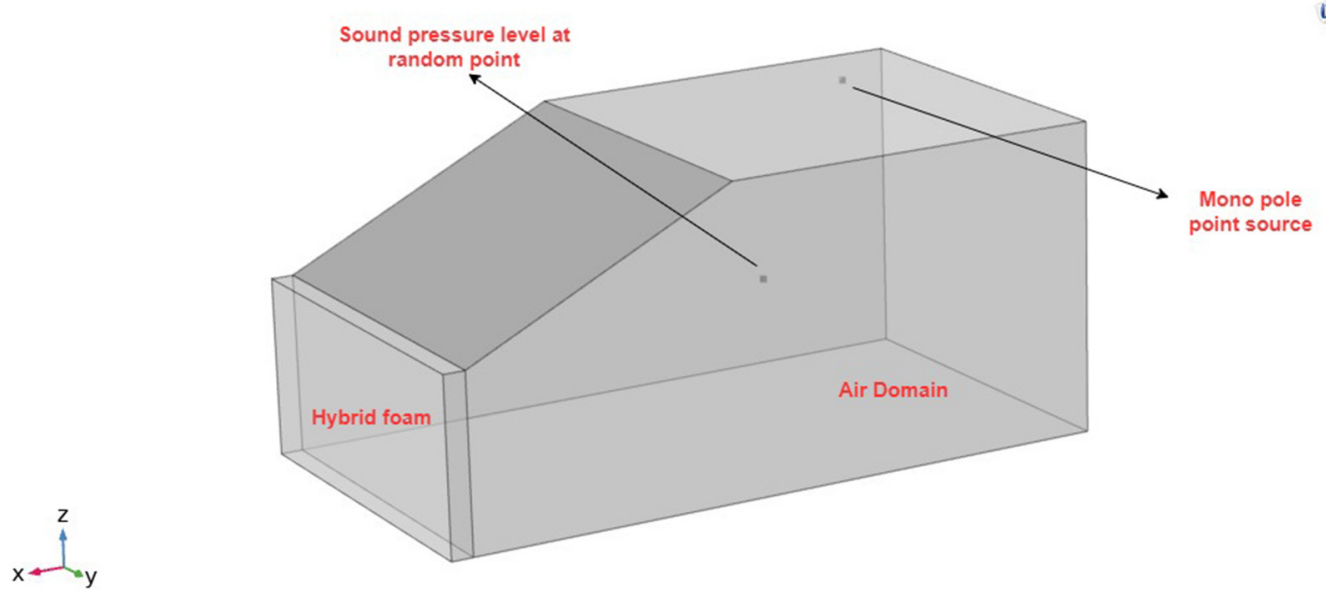

Fig. 5. Design of test bench car coupled with hybrid foam.

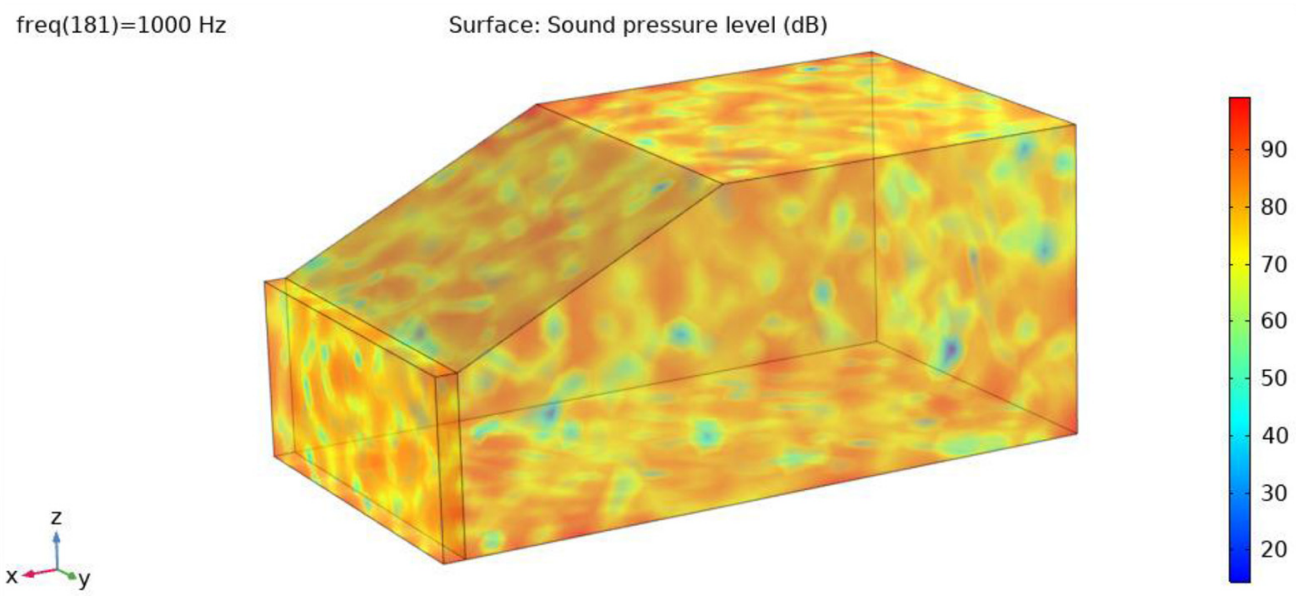

Fig. 6. Numerical simulation of test bench car coupled with hybrid foam.

acoustic boundary layers. Hexahedron element mapped for the whole model and size of the component is chosen based on six elements per wavelength on a higher frequency. For excitation plane wave radiation with unit Pascal is applied. Figure 4 presents the geometry model, mesh model, and simulated model. The material property used for simulation is depicted in Table 5.

\subsubsection{Test bench car simulation}

To examine the effect of the proposed hybrid foam in the car interior, a test bench car simulation was carried out in COMSOL Multiphysics. The dimensions of the test bench car are length $\times$ height $\times$ depth is $3.0 \mathrm{~m} \times 1.4 \mathrm{~m} \times 1.7 \mathrm{~m}$, and the windshield has its lower end $0.8 \mathrm{~m}$ above the floor and with an inclination of $32^{\circ}$ such that the entire volume of the geometry is $6.5 \mathrm{~m}^{3}$. The given dimension resembles the actual car and part body for instance the section which separates engine compartment and cabin is modeled as proposed material because engine noise major source of noise. Based on this front section, hybrid foam is modeled, and better configuration material is applied. A monopole point flow source of strength $Q_{S}=10^{-5} \mathrm{~m}^{3} / \mathrm{s}$ located at the point $P_{0}=(0.21,0$, and 1.28$)$ is chosen. Pressure acoustic frequency domain module applied to the entire model and hybrid foam defined as poro-acoustic material, and five parameter JCA model was chosen from dropdown. The model has meshed with Physics controlled free triangular element with fine size, and the study conducted over the frequency range of $100-1000 \mathrm{~Hz}$. The sound pressure level measured at the point $P_{1}=(1.34,1.22$, and 0.8$)$, geometry model, and SPL simulation is shown in Figures 5 and 6 respectively.

\section{Results and discussion}

\subsection{Experimental sound absorption result}

Figure 7 represents the prepared multilayer configuration's experimental sound absorption coefficient; the metallic foam sample of $40 \mathrm{~mm}$ possesses good sound absorption only at a higher frequency region. This is due to limited 


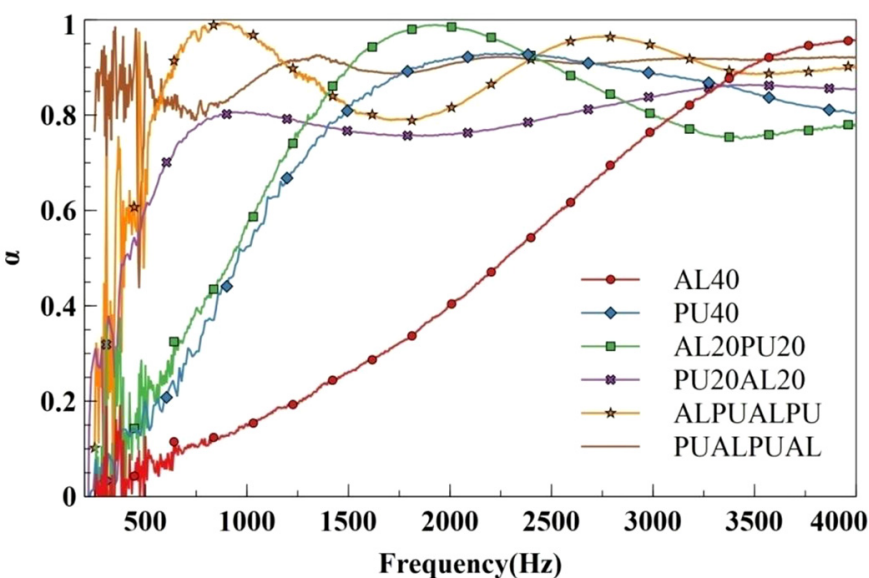

Fig. 7. Results of experimental sound absorption.

viscous losses that too only on the surface of $\mathrm{Al}$ foam, which means higher resistance to the propagation of sound offered by the material. For sample, PU 40 foam sample open cell in nature also exhibits poroelastic property, so the viscous and thermal losses will be comparatively high compared to AL 40, leading to better sound absorption. To enhance sound absorption, this study attempt to produce combination material in a multilayer configuration. $20 \mathrm{~mm} \mathrm{Al} \mathrm{foam}$ kept as face layer followed that $20 \mathrm{~mm}$ PU foam added as an additional layer, now the total configuration AL20PU20 is maintained as $40 \mathrm{~mm}$ thickness. From the tested result, we can notice that sound absorption is slightly resonant behavior and much better than the AL40 sample, also roughly near to the performance of PU40. This is caused by high-density AL20 material acting as mass and low density PU20 act as spring, so the whole configuration possesses additional dampening. By reversing the sample now the configuration is PU20AL20, and its corresponding result indicates that the sound absorption starts well in the lowfrequency region near $500 \mathrm{~Hz}$. The possible reason for this improved sound absorption is low frequency has higher energy, so when it crosses the PU20, half of the sound energy is attenuated, and the rest is reacted with AL20. Further, the configuration is designed with four layers, each value a thickness of $10 \mathrm{~mm}$; now, the sample configuration is ALPUALPU, which resembles the double spring-mass system. Because of this effect, the resonant behavior is identified from the absorption plot, also the peak sound absorption places in the low-frequency region. After reversing the sample now the configuration PUALPUAL is posses better sound absorption among all, the peak sound absorption placed in below $500 \mathrm{~Hz}$, the sound energy attenuated by each layer. Figure 8 represents the validation plot of experimental and numerical results of all samples that possess fair agreement.

\subsection{Test bench car analysis result}

For a simple case study, the better performing configuration sample PUALPUAL corresponding predicted values are used to model the hybrid foam and couple to test bench
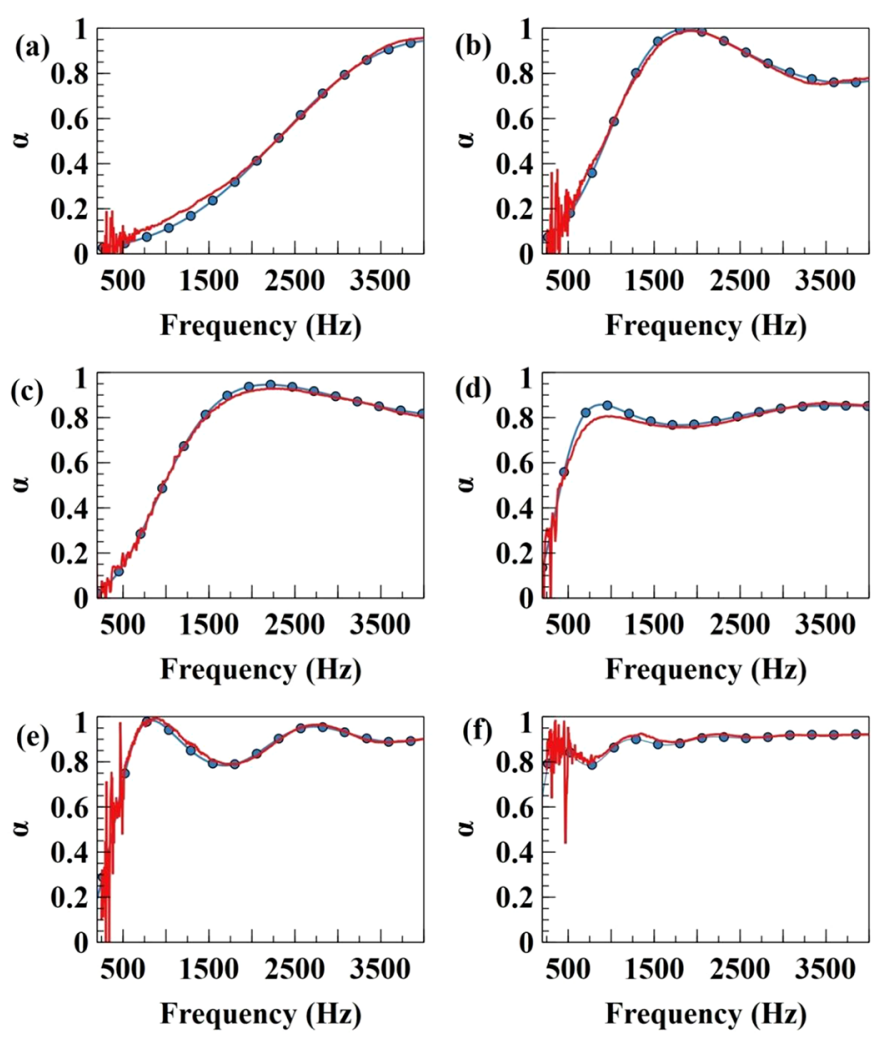

- Experimental

$\multimap \quad$ Numerical

Fig. 8. Validation results of experimental and numerical sound absorption (a) AL 40, (b) PU 40, (c) AL20PU20, (d) PU20AL20, (e) ALPUALPU, (f) PUALPUAL.

car for interior acoustic simulation. The numerical results of test bench simulation with and without hybrid foam are plotted in Figure 9. From the results, it is understood that the proposed configuration suppresses the majority of dominant modes. Further in-depth outlook the result plotted in $1 / 3$ rd octave band in Figure 10, which is mainly used in environmental and noise control application. The octave plots show $5-30 \mathrm{~dB}$ reduction in SPL inside the car cavity.

\section{Conclusion}

The hybrid foam was fabricated by developing a multilayer configuration from $\mathrm{Al}$ (closed cell) and PU (open cell) foam. The measured sound absorption of different layer configurations used to predict the non-acoustic parameters using the PSO algorithm. A simplified numerical model developed in COMSOL Multiphysics to evaluate sound absorption from the predicted parameters. The sound absorption coefficient of the experimental and numerical results are in good agreement. Further, the combination of open-cell and closed-cell foam enhances the sound absorption in a wide range of frequency. Significantly, the $10 \mathrm{~mm}$ layer configuration exhibits better acoustic performance than the 20 and $40 \mathrm{~mm}$ configuration. Once the optimum 


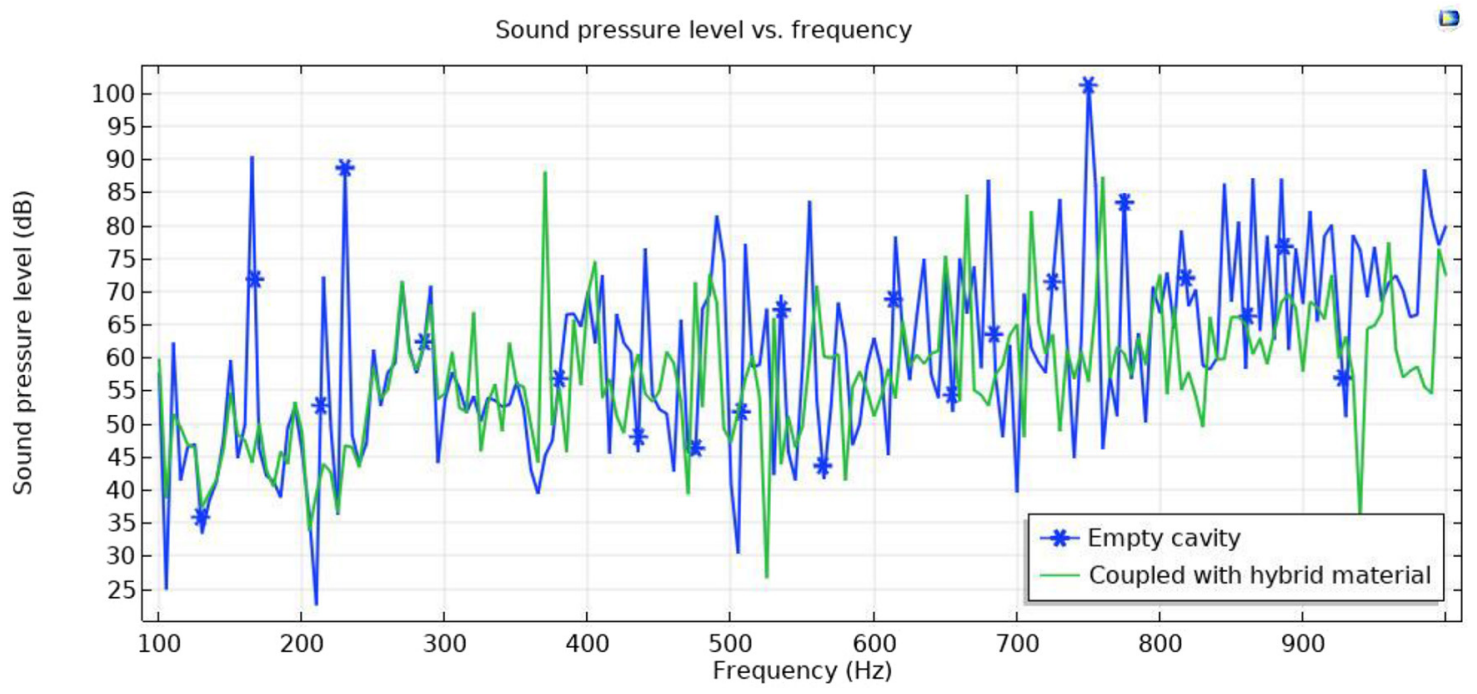

Fig. 9. Result comparison of test bench car with and without hybrid material.

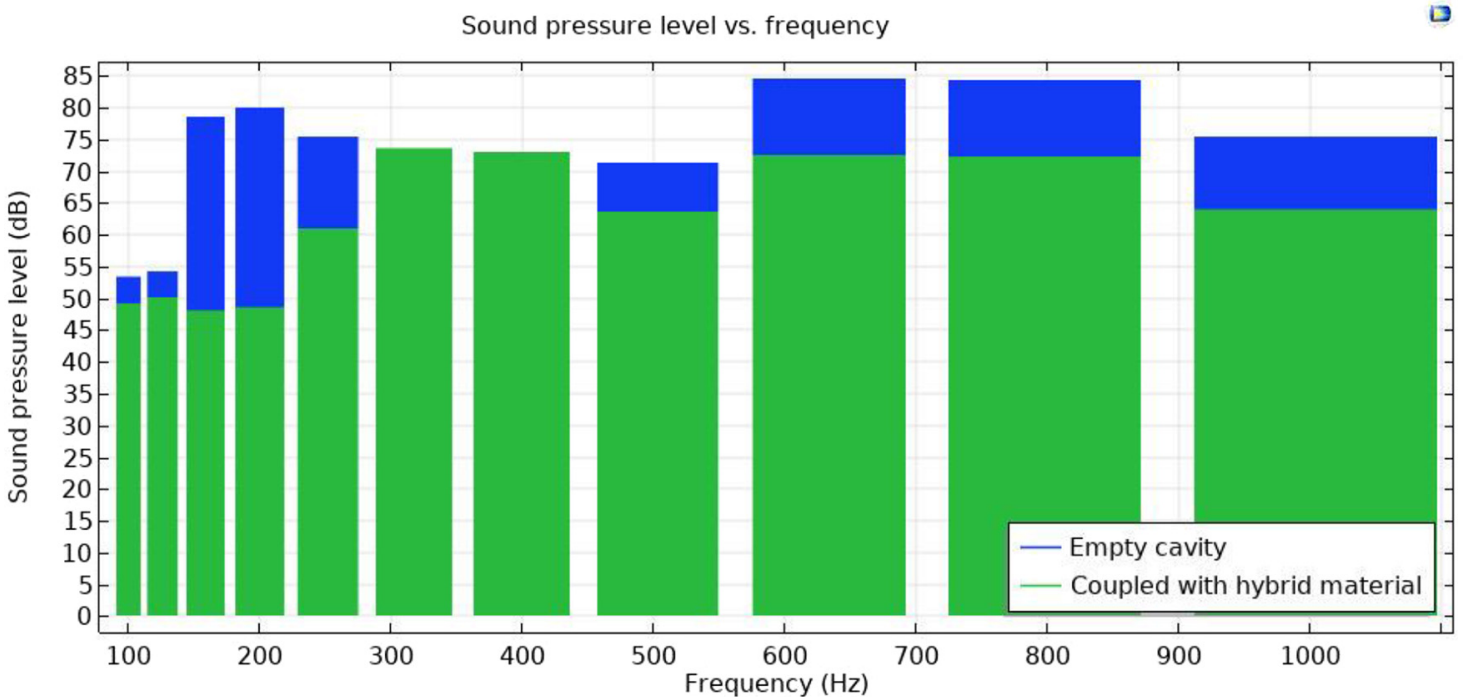

Fig. 10. SPL comparison plot in $1 / 3$ rd octave plot.

performed design is identified and its corresponding predicted value used to model the poro-acoustic material to perform interior acoustic analysis. The sound pressure level of test bench car analysis is compared with and without hybrid foam material. From the numerical results the proposed configuration is capable of noise reduction of $5-30 \mathrm{~dB}$ in $1 / 3$ rd octave band.

\section{References}

1. N. Dukhan, Metal Foams: Fundamentals and Applications, DEStech Publications, Inc. (2013)

2. J.P. Arenas, M.J. Crocker, Recent trends in porous soundabsorbing materials, Sound Vibr. 44, 12-18 (2010)
3. N. Kino, G. Nakano, Y. Suzuki, Non-acoustical and acoustical properties of reticulated and partially reticulated polyurethane foams, Appl. Acoust. 73, 95-108 (2012)

4. O. Doutres, N. Atalla, K. Dong, A semi-phenomenological model to predict the acoustic behavior of fully and partially reticulated polyurethane foams, J. Appl. Phys. 113, 054901 (2013)

5. L. Yuvaraj, S. Jeyanthi, Acoustic performance of countersunk micro-perforated panel in multilayer porous material, Build. Acoust. 27, 3-20 (2020)

6. X. Wang, X. Luo, H. Zhao, Z. Huang, Acoustic perfect absorption and broadband insulation achieved by doublezero metamaterials, Appl. Phys. Lett. 112, 021901 (2018)

7. J. Lardiès, H. Ma, M. Berthillier, Localization of radiating sources by an acoustical array, Int. J. Simul. Multidiscip. Des. Optim. 4, 135-140 (2010) 
8. P. Salzenstein, An example of design, optimization, stabilization and noise performances of resonator-based optoelectronic oscillators, Int. J. Simul. Multidiscip. Des. Optim. 10, A2 (2019)

9. P.V. Bansod, A.R. Mohanty, Inverse acoustical characterization of natural jute sound absorbing material by the particle swarm optimization method, Appl. Acoust. 112, 41-52 (2016)

10. L. Yuvaraj, S. Jeyanthi, A. Yogananda, An acoustical investigation of partial perforation in jute fiber composite panel, Mater. Today: Proc. (2020)
11. L. Yuvaraj, S. Jeyanthi, L.B. MailanChinnapandi, Experimental and finite element approach for finding sound absorption coefficient of bio-based foam, J. Vibroeng. 21, 1761-1771 (2019)

12. R. Sailesh, L. Yuvaraj, J. Pitchaimani, M. Doddamani, L.B.M. Chinnapandi, Acoustic behaviour of 3D printed biodegradable micro-perforated panels with varying perforation cross-sections, Appl. Acoustics 174, 107769 (2021)

13. ASTM Standard E1050-12, Standard test method for impedance and absorption of acoustical materials using a tube, two microphones, and a digital frequency analysis system

Cite this article as: L. Yuvaraj, S. Jeyanthi, Lenin Babu Mailan Chinnapandi, Elammaran Jayamani, Design and simulation of multilayer hybrid foam material for acoustic application, Int. J. Simul. Multidisci. Des. Optim. 12, 10 (2021) 